\title{
A Study of MANET Routing Protocols: Joint Node Density, Packet Length and Mobility
}

\author{
Nurul I. Sarkar \\ School of Computing and Mathematical Sciences \\ Auckland University of Technology \\ Auckland, New Zealand \\ nurul.sarkar@aut.ac.nz
}

\author{
Wilford G. Lol \\ School of Computing and Mathematical Sciences \\ Auckland University of Technology \\ Auckland, New Zealand \\ wilfordlol@gmail.com
}

\begin{abstract}
The dynamic topology of a mobile ad hoc network (MANET) poses a real challenge in the design of a MANET routing protocol. Over the last 10 years, a variety of routing protocols have been developed and their performance simulations are made by network researchers. Most of the previous research on MANET routing protocols have focused on simulation study by varying network parameters, such as network size (node density), pause times, or node mobility independently. This paper considers the problem from a different perspective, using a simulation model the combined effect of node density and packet length; node density and mobility on the performance of a typical 802.11 MANET is investigated. This is a common and realistic scenario in MANETs where nodes move around, join and leave the network at any time. Based on the QoS (end-to-end delay, throughput), routing load and packet retransmissions, this paper systematically analyzes the performance of four diverse MANET routing protocols with the different simulation model and configurations, and drew more complete conclusions.
\end{abstract}

Keywords: Mobile ad hoc networks, node density, routing protocols

\section{INTRODUCTION}

A mobile ad hoc network (MANET) is a collection of wireless mobile nodes that are communicating with each other using multi-hop wireless links without a centralized network infrastructure. Such networks are being deployed for many diverse applications, such as military network environments in the battle fields, disaster operations, search and rescue operations, conference rooms [1], and also in commercial applications such as vehicle ad hoc networks used in taxi service operation [2].

Because the nodes in a MANET are mobile, the physical network topology changes frequently and unpredictably. In MANETs, there is no stationary infrastructure such as access points (APs), therefore each node has to act as router for forwarding packets to other nodes.

Over the last 10 years, various MANET routing protocols have been developed by network researchers and designers primarily to improve the MANET performance with respect to establishing correct and efficient routes between a pair of nodes for packet delivery. Examples of popular MANET routing protocols are: Optimized Link State Routing (OLSR) [3, 4]. Ad
Hoc On-Demand Distance Vector (AODV) [1, 5], Dynamic Source Routing (DSR) [6, 7], and Temporally Ordered Routing Algorithm (TORA) [8, 9].

OLSR is a proactive (table-driven) routing protocol, finds routing paths in advance for all source and destination pairs and periodically exchange topology information to maintain them. AODV, DSR, and TORA are reactive routing protocols, share the on-demand behaviors and find the route only when packets to be transmitted. However, the routing mechanisms for these reactive protocols are quite different. For example, AODV uses table-driven approach and destination sequence numbers. DSR uses a source initiated routing, whereas TORA uses a link reversal routing mechanism. More details about OLSR can be found in networking literature [7, 10], and AODV, DSR and TORA in $[11,12]$.

Performance of a MANET routing protocol depends on various factors, including the complex interplay of protocol mechanisms and their specific settings with node density, mobility, packet length (i.e. traffic intensity) and the behavior of wireless nodes used. A good understanding of the joint effect of node density, packet length and mobility on the performance of a typical IEEE 802.11 ("802.11") MANET is required for an efficient design and deployment of such systems. This paper investigates the combined effect of node density and packet length; node density and mobility on the performance of four different MANET routing protocols.

In this paper we address the following research question: What impact do different routing protocols (OLSR, AODV, DSR, and TORA) have on a typical 802.11 MANET for varying node density and packet length; node density and mobility concurrently?

To answer the question posed we carry out a systematic performance analysis (by simulation) for four typical MANET routing protocols, which include one proactive routing protocol OSLR, and three on-demand routing protocols, AODV, DSR, and TORA. These routing protocols were selected based on their popularity, published results, and interesting characteristics and features.

The remainder of this paper is structured as follows. Section II reviews literature, representative of that MANET routing protocols. Section III describes simulation environment and parameters. The simulation results and comparative analysis of four routing protocols are presented in Section IV. 
The system implication is discussed in Section V and Section VI concludes the paper.

\section{LITERATURE REVIEW}

To improve the performance of a typical MANET, various routing protocols have been proposed by many network researchers. For brevity only a selected set of literature that is indicative of the range of approaches used for improving and analyzing MANET routing performance is reported in this section.

Sjaugi et al. [13] proposed a route maintenance mechanism for DSR called DISTANCE. The key idea is to introduce a special node called "bridge node" into the source list for preventing link failures. Each node updates its location by piggybacking into packet header. By preventing link failure, the system improves packet sending ratios and delays.

Taing et al. [6] proposed an improved DSR called Modified DSR (MDSR). MDSR provides better delays and number of hop paths from source to destination than DSR. The idea is to select a shortest path for delay-sensitive traffic using larger power level. For delay insensitive traffic, MDSR uses smaller power level.

Lu et al. [14] developed a MANET routing protocol called Congestion Aware Distance Vector (CADV) to improve network performance in terms of packet delivery and routing load. CADV integrates congestion avoidance strategy into a proactive routing protocol such as Destination Sequence Distance Vector (DSDV).

Wei et al. [15] proposed a routing protocol called Power Control AODV (PC-AODV) to improve network throughput and power consumption. The idea is to establish a route with an appropriate data-rate link within the transmission range and to adjust the transmit power level.

Safa et al. [16] proposed a routing protocol called Heterogeneous AODV (HAODV), optimizes existing AODV to support routing in heterogeneous networks (e.g. Wi-Fi and Bluetooth nodes). HAODV determines an optimum route not only based on path with the lowest hop-count but also with the low traffic and high stability.

Bai et al. [17] developed a routing protocol called DOA (DSR over AODV), focusing on route maintenance. DOA implements two levels of route repair: intra-segment and intersegment. If a route fails, an intra-segment fixes it by using alternative routes within one segment.

Khamforoosh et al. [18] proposed another class of AODV called Centre base Distance Multi-path AODV (CDM-AODV). The idea is to choose two paths from the centre of the network. The reason being is that there is a reverse relationship between the distances of the node to the centre of network. When request packets are sent, replay packets have the information about the centre of network and distance between nodes.

In addition to the above proposals, earlier works on MANETs focused on routing performance comparison by extensive simulations. For example, Das et al. [19, 20] evaluated MANET routing protocols by simulation with varying nodes and pause times. Biao [21] examined the performance of DSDV, AODV, DSR and TORA by extensive simulations with increasing the number of nodes in MANETs.

The MANET routing approaches reviewed in this section are grouped into three main categories shown in Table I.
TABLE I. CATEGORIES OF ROUTING APPROACHES REVIEWED

\begin{tabular}{|l|l|}
\hline Approaches & Example of routing protocols \\
\hline $\begin{array}{l}\text { Exchanging location information } \\
\text { by piggybacking. }\end{array}$ & DISTANCE [13]. \\
\hline $\begin{array}{l}\text { Selecting shortest path using } \\
\text { power level, adjusting transmit } \\
\text { power, and route optimization. }\end{array}$ & $\begin{array}{l}\text { MDSR [6], PC-AODV [15], } \\
\text { AODV [18]. }\end{array}$ \\
\hline $\begin{array}{l}\text { Implementing a congestion } \\
\text { avoidance strategy in DSDV. }\end{array}$ & CADV [14]. \\
\hline
\end{tabular}

\section{SIMULATION ENVIRONMENT AND PARAMETERS}

\section{A. Modeling the network and simulation parameters}

We use OPNET Modeler 15.0 [23] to analyze the performance of OLSR, AODV, DSR and TORA. In the simulations we consider three network scenarios: (1) a small network with $\mathrm{N} \leq 10$ nodes; (2) a medium sized network with $10>\mathrm{N} \leq 50$ nodes; and (3) a denser network with $50>\mathrm{N} \leq 100$ nodes. The mobile nodes are placed randomly within a $2000 \mathrm{~m}$ $\times 2000 \mathrm{~m}$ area. Radio propagation range for each node is 250 $\mathrm{m}$ and channel capacity is $11 \mathrm{Mbps}$. Each node moves in this area according to the random waypoint mobility model, with a speed of 20 to $30 \mathrm{~m} / \mathrm{s}$ and no pause time, which means that nodes are always moving in the entire simulation period.

Tables II to VI list the parameter values used in the simulations. Each simulation run lasted for 900 seconds simulated time in which the first 10 seconds was the transient period. The observations collected during the transient period are not included in the final simulation results.

The four performance metrics, such as end-to-end packet delay, throughput, routing load and retransmission were used for performance study of OLSR, AODV, DSR, and TORA. The end-to-end packet delay is defined as the average time (measured in seconds) required in sending a packet from source to a destination. This includes buffering during route discovery, queuing at the interface queue, retransmission at the medium access control (MAC), propagation and packet transmission time. The throughput (measured in bps) is the average rate of successful packet delivery. The routing load is the number of routing control packets transmitted for each data packet delivered at the destination. The retransmission is defined as the resending attempts of packets which have been lost or damaged due to link failure.

TABLE II. GENERAL PARAMETERS USED IN SIMULATION

\begin{tabular}{|c|c|}
\hline Parameter & Value \\
\hline Simulation area & $2000 \mathrm{~m} \times 2000 \mathrm{~m}$ \\
\hline Node density & $10,50,100$ nodes \\
\hline Packet length & $1,000,5,000$ and 50,000 bytes \\
\hline Node mobility & $5,20,30(\mathrm{~m} / \mathrm{s})$ \\
\hline Mobility model & Random waypoint with 0 pause time \\
\hline Wireless cards & $802.11 \mathrm{~b}(11 \mathrm{Mbps})$ \\
\hline RTS/CTS & OFF \\
\hline Propagation range & $0.005 \mathrm{Watt}$ \\
\hline Transmitter power & FTP \\
\hline Traffic/application & TCP \\
\hline Transport protocol & 900 seconds \\
\hline Simulation duration &
\end{tabular}


TABLE III. OLSR PARAMETERS USED IN SIMULATION.

\begin{tabular}{|c|c|}
\hline Parameter & Value \\
\hline Willingness & default \\
\hline Hello interval & $2 \mathrm{~s}$ \\
\hline TC Interval & $5 \mathrm{~s}$ \\
\hline Neighbour hold time & $6 \mathrm{~s}$ \\
\hline Topology hold time & $15 \mathrm{~s}$ \\
\hline
\end{tabular}

TABLE IV. AODV PARAMETERS USED IN SIMULATION.

\begin{tabular}{|c|c|}
\hline Parameter & Value \\
\hline Route request retries (route discovery) & 5 \\
\hline Route request rate & 10 (packets/s) \\
\hline HELLO interval (uniform) & Between 1 and $1.1 \mathrm{~s}$ \\
\hline Route error rate & 10 packets/s \\
\hline Node traversal time & $0.04 \mathrm{sec}$ \\
\hline Timeout buffer & 2 \\
\hline Local repair & enabled \\
\hline
\end{tabular}

TABLE V. DSR PARAMETERS USED IN SIMULATION.

\begin{tabular}{|l|l|}
\hline Parameter & Value \\
\hline Request table size (nodes) (route discovery) & 64 \\
\hline Max Request retransmission (route discovery) & 16 \\
\hline Max request period (sec) route discovery & 10 \\
\hline Max buffer size for route maintenance & 50 packets \\
\hline Maintenance hold time & $0.25 \mathrm{~s}$ \\
\hline Max maintenance retransmission & 2 \\
\hline Maintenance acknowledgement timer & $0.5 \mathrm{~s}$ \\
\hline Route replies using cached routes & enabled \\
\hline Packet salving & enabled \\
\hline
\end{tabular}

TABLE VI. TORA PARAMETERS USED IN SIMULATION.

\begin{tabular}{|l|l|}
\hline Parameter & Value \\
\hline Mode of operation & On-demand \\
\hline OPT transmission interval & $300 \mathrm{sec}$ \\
\hline IP Packet discard timeout & $10 \mathrm{sec}$ \\
\hline Beacon period & $20 \mathrm{sec}$ \\
\hline Max Beacon timer & $60 \mathrm{sec}$ \\
\hline Max tries (number of attempts) & 3 \\
\hline
\end{tabular}

\section{B. Model Validation}

A credible network simulator may produce invalid results if the simulation parameters are not correctly configured. Therefore, simulation model validation becomes an important part of any simulation study. The OPNET simulation model presented in this paper was verified in several ways. First, the detailed status information including mobility traces was observed throughout the simulation to verify the model. Second, we checked the level of network fragmentation for 10 nodes with $250 \mathrm{~m}$ transmission range distributed over a square of $2000 \mathrm{~m} \times 2000 \mathrm{~m}$. In addition, OPNET results were compared with the results obtained from ns-2 [24] and a good match between two sets of results further validated the simulation models.

\section{RESULTS AND COMPARATIVE ANALYSIS}

To investigate the combined effect of node density and packet length, and node density and mobility for OLSR, AODV, DSR, and TORA on an 802.11 MANET (RTS/CTS $\mathrm{OFF})$, we consider three node density scenarios: $\mathrm{N}=10,50$, and 100 nodes; and two node mobility scenarios: 20 and $30 \mathrm{~m} / \mathrm{s}$. The data packet length of 1,000,5,000, and 50,000 bytes were used in the simulations. All simulation results report the network steady state and were obtained with a relative statistical error $\leq 1 \%$, at $99 \%$ confidence level.

\section{A. Joint effect of node density and packet length}

The combined effect of node density $(\mathrm{N}=10,50$, and 100 nodes) and packet length $(5,000,50,000$ bytes) on mean packet delay for OLSR, AODV, DSR, and TORA is illustrated in Fig. 1. The mean packet delay is higher for 50,000-byte packets than for 5,000-byte packets for DSR and AODV at both $\mathrm{N}=50$ and 100 nodes, but not for OLSR and TORA. TORA achieves slightly lower packet delays under high traffic loads (50,000-byte packets) at $\mathrm{N}=100$ nodes. Overall, OLSR achieved the lowest packet delays than the other three routing protocols examined at $\mathrm{N}=100$ nodes.

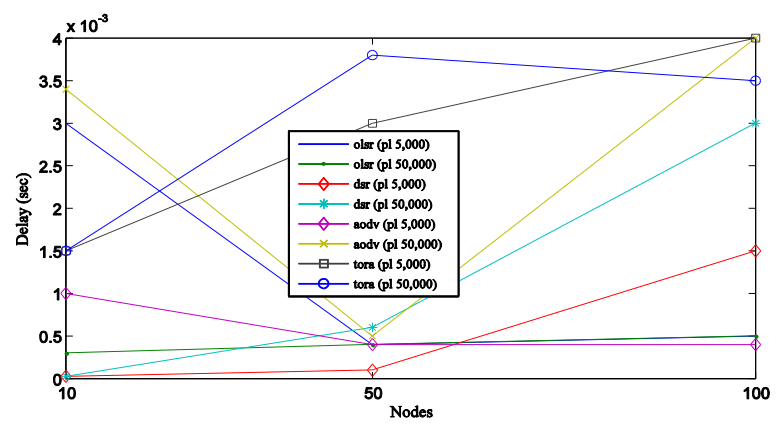

Figure 1. Packet delay versus node density for packet lengths of 5,000 and 50, 000 bytes (node mobility: $5 \mathrm{~m} / \mathrm{s}$ ).

The combined effect of node density and packet length on network throughput for OLSR, AODV, DSR, and TORA is demonstrated in Fig. 2. The network throughput is higher for 50,000-byte packets than for 5,000-byte packets for DSR and TORA at $\mathrm{N}=100$ nodes, but not for OLSR and AODV. TORA achieved the highest throughput than the other three routing protocols at $\mathrm{N}=100$ nodes. Overall, the network throughput increases with the number of active nodes for all four routing protocols studied.

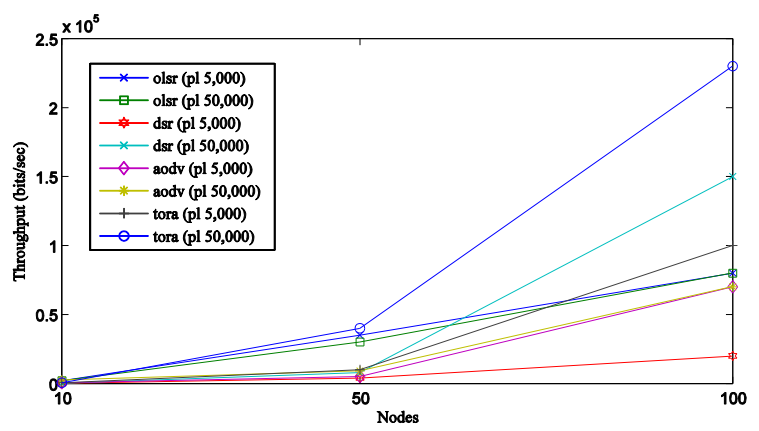

Figure 2. Throughput versus node density for packet lengths of 5,000 and 50,000 bytes (node mobility: $5 \mathrm{~m} / \mathrm{s}$ ). 
The combined effect of node density and packet length on routing load for OLSR, AODV, DSR, and TORA is illustrated in Fig. 3. The routing load characteristics for all four routing protocols are similar to the network throughput performance discussed earlier (Fig. 2). OLSR maintains a low and consistent routing load at $\mathrm{N}=100$ nodes.

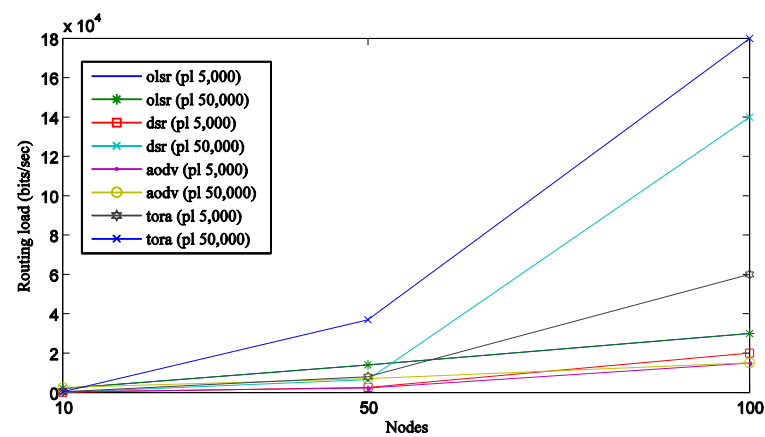

Figure 3. Routing load versus node density for packet lengths of 5,000 and 50,000 bytes (node mobility: $5 \mathrm{~m} / \mathrm{s}$ ).

The combined effect of node density and packet length on packet retransmission attempts for OLSR, AODV, DSR, and TORA is illustrated in Fig. 4. The packet retransmission is higher for 50,000-byte packets than for 5,000-byte packets for DSR and TORA at $\mathrm{N}=100$ nodes, but not for OLSR and AODV. TORA has higher retransmission attempts than DSR for 50,000-byte packets at $\mathrm{N}=100$ nodes. Another observation is that TORA has slightly lower packet retransmissions under high traffic loads at $\mathrm{N}=50$ nodes.

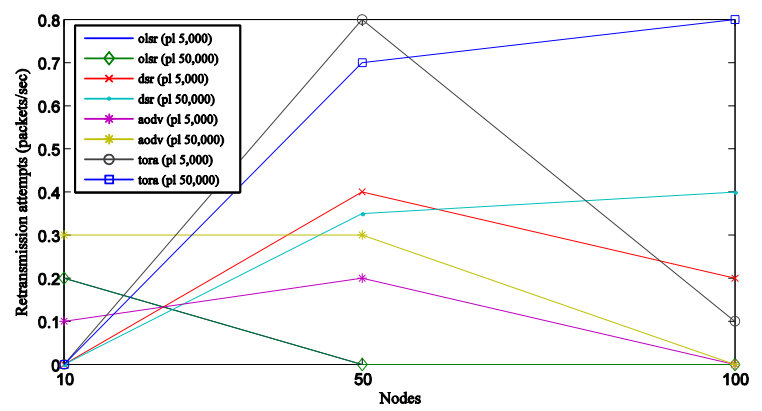

Figure 4. Retransmission versus node density for packet lengths of 5,000 and 50,000 bytes (node mobility: $5 \mathrm{~m} / \mathrm{s}$ ).

\section{B. Joint effect of node density and mobility}

The combined effect of node density $(\mathrm{N}=10,50$, and 100 nodes) and node mobility (20 and $30 \mathrm{~m} / \mathrm{s}$ ) on mean packet delay for OLSR, AODV, DSR, and TORA is illustrated in Fig. 5. The packet delay increases when the number of nodes increases from 50 to 100 for all routing protocols examined.

Of the four routing protocols, TORA has high packet delays especially for a large network with high mobility. OLSR achieves shorter delays because it is a kind of proactive routing protocol where each node maintains a routing table with possible destinations and the number of hops to each destination. When a packet arrives at a node; it is either forwarded immediately or dropped off.

AODV uses the source-initiated approach in the route discovery process, but for route maintenance it uses tabledriven mechanism. AODV performs better (in terms of packet delays) than DSR when node mobility is high. Our findings are in close agreement with the work of other researchers [20].

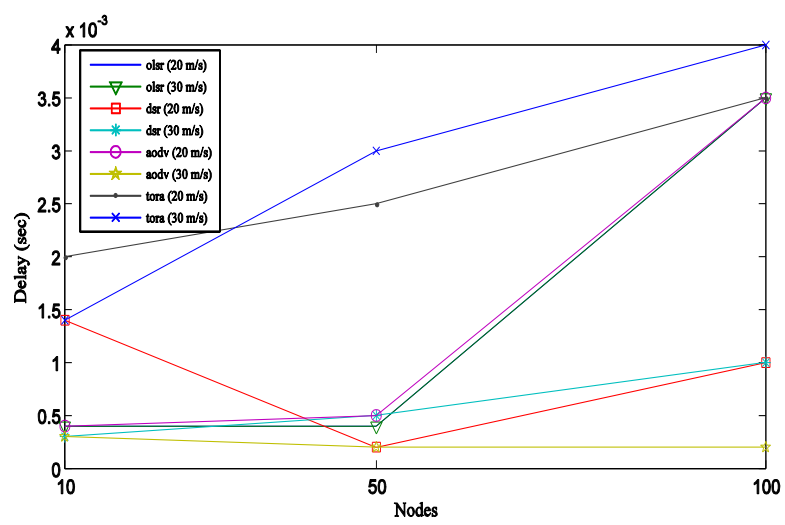

Figure 5. Packet delay versus node density for mobility 20 and $30 \mathrm{~m} / \mathrm{s}$.

The combined effect of node density and node mobility on network throughput for OLSR, AODV, DSR, and TORA is illustrated in Fig. 6. The network throughput increases quickly for TORA, OLSR, and AODV with increased node density and mobility.

DSR on the other hand has difficulties in finding routes when both node density and mobility increase, throughput drops slightly for the node density fewer than 50 nodes. However, OLSR achieves slightly better throughput than the other reactive protocols (TORA, AODV, and DSR) for a small to medium sized network. This is because OLSR senses neighboring nodes to establish a connection and finds a valid route. AODV reacted in the same way as TORA; however the high node mobility reduces network throughputs. TORA offers better throughput for a large network with high mobility than AODV and DSR.

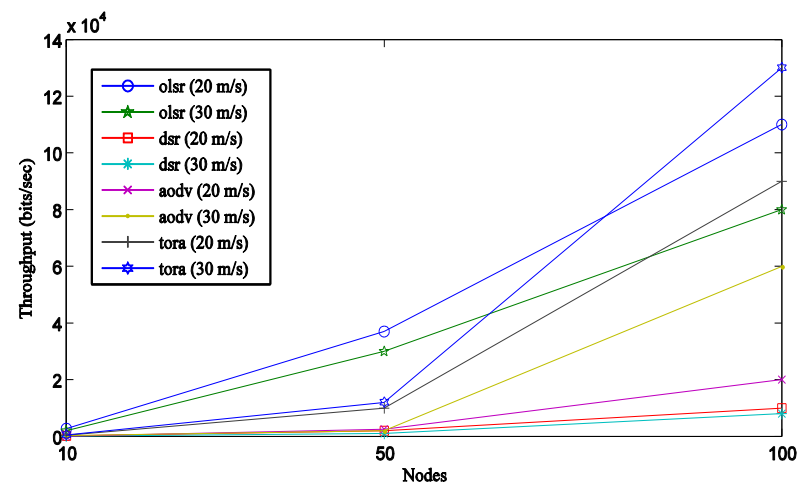

Figure 6. Throughput versus node density for mobility 20 and $30 \mathrm{~m} / \mathrm{s}$.

The combined effect of node density and node mobility on routing load for OLSR, AODV, DSR, and TORA is illustrated in Fig. 7. The routing load of a protocol can influence node's 
efficiency of battery energy and scalability. The four routing protocols have different routing overhead. For example, OLSR has higher routing load than AODV, DSR, and TORA for node density fewer than 50 nodes. However, in the case of a large network (around 100 nodes), OLSR has slightly smaller routing load than TORA. DSR achieved the lightest routing load than other three routing protocols.

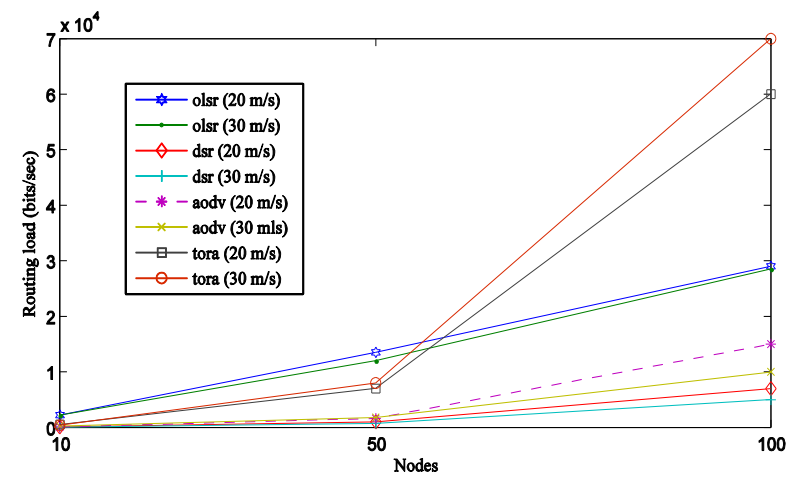

Figure 7. Routing load versus node density for mobility 20 and $30 \mathrm{~m} / \mathrm{s}$.

The combined effect of node density and mobility on packet retransmission attempts for OLSR, AODV, DSR, and TORA is demonstrated in Fig. 8. Of the four routing protocols, OLSR has the least packet retransmission attempts, whereas TORA has the highest retransmission rate for all network scenarios. However, AODV's packet retransmission rate is slightly lower than DSR especially for a large network with more than 50 nodes and high mobility.

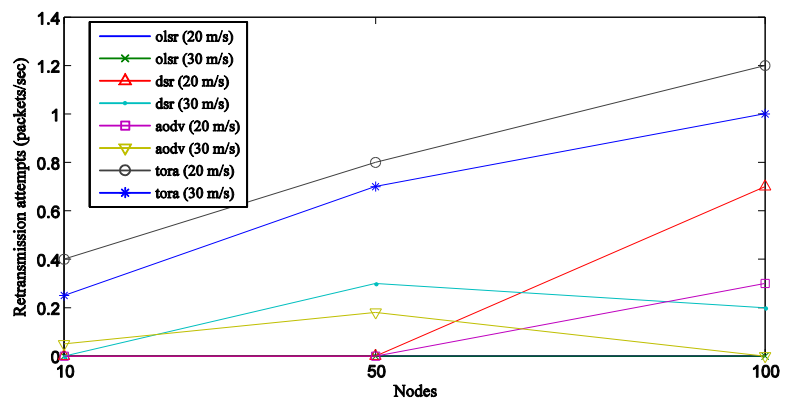

Figure 8. Retransmission versus node density for mobility 20 and $30 \mathrm{~m} / \mathrm{s}$.

\section{DISCUSSION AND PRACTICAL IMPLICATIONS}

The results presented in Section IV provide some insight into the combined effect of node density, packet length and mobility for OSLR, AODV, DSR, and TORA on the performance of a typical 802.11 MANET. Empirical results show that the combined node density and mobility has a significant effect on network throughput and packet delays for all four routing protocols studied. However, the effect of packet length has minor effects on network throughput and packet delays specifically for selected routing protocols (Figs. 1 and 2).

For a network of up to 10 active nodes with low mobility, all four routing protocols performed well to a certain degree.
However, as the number of nodes increases to 100 , there is a significant variation in throughputs obtained by each of the four routing protocols. For example, both OLSR and TORA performed well (in terms of throughput) especially for a network with 50 to 100 nodes. TORA reacted favorably to node mobility; achieves better throughput with high mobility. AODV reacted in the same way as TORA; however, the high node mobility reduces its throughput. TORA's packet delay deteriorates with high node mobility.

From a practical networking point of view, a question may arise about the right routing protocol to use for a particular network scenario.

Table VII lists the six network scenarios and the corresponding MANET routing protocol to use for best system performance. For instance, for a denser network with $\mathrm{N}=100$ nodes moving at high speed $(30 \mathrm{~m} / \mathrm{s})$ under high traffic loads, TORA is the best routing protocol to use. For another scenario where a small network with fewer than 10 nodes moving at medium speed $(20 \mathrm{~m} / \mathrm{s})$ under low loads, OLSR is the best routing protocol to use.

TABLE VII. NETWORK SCENARIOS AND THE BEST PROTOCOL TO USE.

\begin{tabular}{|c|c|}
\hline Scenario & Best protocol to use \\
\hline $\begin{array}{l}\text { 1. Small network with low traffic and } \\
\text { medium mobility }\end{array}$ & OSLR, TORA \\
\hline $\begin{array}{l}\text { 2. Small network with high traffic and } \\
\text { high mobility }\end{array}$ & AODV, OLSR \\
\hline $\begin{array}{l}\text { 3. Medium network with low traffic and } \\
\text { medium mobility }\end{array}$ & \multirow{3}{*}{ TORA, OLSR } \\
\hline $\begin{array}{l}\text { 4. Medium network with high traffic and } \\
\text { high mobility }\end{array}$ & \\
\hline $\begin{array}{l}\text { 5. Large network with low traffic and } \\
\text { medium mobility }\end{array}$ & \\
\hline $\begin{array}{l}\text { 6. Large network with high traffic and } \\
\text { high mobility }\end{array}$ & TORA, DSR \\
\hline \multicolumn{2}{|c|}{$\begin{array}{l}\text { Network -> Small: } 10 \text { nodes; medium: } 50 \text { nodes; large: } 100 \text { nodes. } \\
\text { Mobility } \rightarrow \text { medium: } 20 \mathrm{~m} / \mathrm{s} \text {; high: } 30 \mathrm{~m} / \mathrm{s} \\
\text { Low traffic } \rightarrow \text { Packet length: } 1000 \text { bytes; High traffic-> Packet } \\
\text { length: } 50,0000 \text { bytes. }\end{array}$} \\
\hline
\end{tabular}

\section{CONCLUSIONS}

The combined effect of node density, packet length and mobility for four routing protocols (OLSR, AODV, DSR, and TORA) on an 802.11 MANET is investigated in this paper. We developed OPNET-based simulation models to study the performance of OLSR, AODV, DSR, and TORA for small, medium and large (dense) network scenarios with varying packet length and node mobility.

Simulation results obtained show that node density and mobility has a significant impact on underlying routing protocols. None of the protocols investigated can offer an optimum routing solution under various network scenarios. For example, OLSR and DSR work well in a small network (fewer than 10 nodes) with low mobility. For a medium-sized network (around 50 nodes) with node mobility, OLSR and AODV offer better performance. In a large network with 100 nodes, TORA and OLSR can offer better performance.

To provide an optimum MANET routing solution, we are currently implementing an efficient MANET routing protocol in OPNET Modeler 15.0 and a future paper will report the projected performance. 


\section{REFERENCES}

[1] H. Pucha, S. M. Das, and Y. C. Hu, "The performance impact of traffic patterns on routing protocols in mobile ad hoc networks," Computer Networks, vol. 51, no. 12, pp. 3595-3616, 2007.

[2] E. Huang, W. Hu, J. Crowcroft, and I. Wassell, "Towards commercial mobile ad hoc network applications: a radio dispatch system," presented at the 6th ACM International Symposium on Mobile Ad Hoc Networking and Computing, 2005, pp. 355-365.

[3] A. Huhtonen, "Comparing AODV and OLSR routing protocols," presented at Telecommunications Software and Multimedia, 2004, pp. 19.

[4] M. Benzaid, P. Minet, and K. Al Agha, "Integrating fast mobility in the OLSR routing protocol," presented at the fourth IEEE Conference in Mobile and Wireless Communications 2002, pp. 1-5.

[5] G. Jayakumar and G. Gopinath, "Performance comparison of two ondemand routing protocols for ad-hoc networks based on random way point mobility model," American Journal of Applied Sciences, vol. 5, no. 6, pp. 659-664, 2008.

[6] N. Taing, S. Thipchaksurat, R. Varakulsiripunth, and H. Ishii, "Performance improvement of dynamic source routing protocol for multimedia services in mobile ad hoc network," presented at the 1st International Symposium on Wireless Pervasive Computing, 2006, pp. 15 .

[7] F. D. Rango, J.-C. Cano, M. Fotino, C. Calafate, P. Manzoni, and S. Marano, "OLSR vs DSR: A comparative analysis of proactive and reactive mechanisms from an energetic point of view in wireless ad hoc networks," Computer Communications, vol. 31, no. 16, pp. 3843-3854, 2008.

[8] S. Giannoulis, C. Antonopoulos, E. Topalis, and S. Koubias, "ZRP versus DSR and TORA: A comprehensive survey on ZRP performance," presented at the 10th IEEE Conference on Emerging Technologies and Factory Automation (ETFA '05), 2005, pp. 1-8

[9] F. Yu, Y. Li, F. Fang, and Q. Chen, "A new TORA-based energy aware routing protocol in mobile ad hoc networks," presented at the 3rd IEEE/IFIP International Conference in Central Asia on Internet, ICI '07, 2007, pp. 1-4.

[10] J. Costa-Requena, T. Vadar, R. Kantola, and N. Beijar, "AODV-OLSR scalable ad hoc routing proposal," presented at the 1st International Symposium on Wireless Pervasive Computing, 2006, pp. 1-5.

[11] E. M. Royer and C. K. Toh, "A review of current routing protocols for ad hoc mobile wireless networks," IEEE Personal Communications, vol. 6, no. 2, pp. 46-55, 1999.

[12] C. E. Perkins, E. M. Royer, S. R. Das, and M. K. Marina, "Performance comparison of two on-demand routing protocols for ad hoc networks," IEEE Personal Communications, vol. 8, no. 1, pp. 16-28, 2001.
[13] M. F. Sjaugi, M. Othman, M. Rasid, and A. Fadlee, "A new route maintenance strategy for dynamic source routing protocol," presented at the International Conference on Information Networking (ICOIN'08) 2008, pp. 1-4.

[14] L. Yi, W. Weichao, Z. Yuhui, and B. Bhargava, "Study of distance vector routing protocols for mobile ad hoc networks," presented at the First IEEE International Conference on Pervasive Computing and Communications (PerCom 2003), March 23-26, 2003, pp. 187-194.

[15] W. Yifei, S. Mei, and S. Junde, "An AODV-improved routing based on power control in WiFi mesh networks," presented at Canadian Conference on Electrical and Computer Engineering (CCECE '08), Niagara Falls, Canada, May 4-7, 2008, pp. 1-4.

[16] H. Safa, H. Artail, M. Karam, H. Ollaic, and R. Abdallah, "HAODV: a New Routing Protocol to Support Interoperability in Heterogeneous MANET," presented at the IEEE/ACS International Conference on Computer Systems and Applications (AICCSA '07), May 13-16 2007, pp. 893-900.

[17] R. Bai and M. Singhal, "DOA:DSR over AODV routing for mobile ad hoc networks," IEEE Transactions on Mobile Computing, vol. 5, no. 10, pp. 1403-1416, 2006.

[18] K. Khamforoosh, A. M. Rahmani, and A. Sheikh Ahmadi, "A new multipath AODV routing based on distance of nodes from the network center," presented at the Mosharaka International Conference on Communications, Propagation and Electronics (MIC-CPE '08), 2008, pp. $1-5$.

[19] S. R. Das, R. Castaneda, and J. Yan, "Simulation-based performance evaluation of routing protocols for mobile ad hoc networks," Mobile Networks and Applications, vol. 5, no. 1, pp. 179-189, 2000.

[20] S. R. Das, C. E. Perkins, and E. M. Royer, "Performance comparison of two on-demand routing protocols for ad hoc networks," presented at the Nineteenth Annual Joint Conference of the IEEE Computer and Communications Societies (INFOCOM '00), Tel Aviv, Israel, March 2000, pp. 3-12.

[21] Q. Biao, H. Jianhua, and Y. Zongkai, "Simulation of wireless Ad hoc routing protocols and its evaluation," Huazhong University of Science and Technology Nature Science (Natural Science Edition), vol. 32, no. 8, pp. 66-69, 2004.

[22] R. Bai and M. Singhal, "DOA: DSR over AODV routing for mobile ad hoc networks," IEEE Transactions on Mobile Computing, no. 14031416, 2006.

[23] OPNET Modeler. Retrieved July 20, 2009, from www.opnet.com

[24] M. Greis. Ns-2 tutorial. Retrieved July 20, 2009, from http://www.isi.edu/nsnam/ns/tutorial/index.html 